\title{
SYMMETRIC POWERS OF COMPLETE MODULES OVER A TWO-DIMENSIONAL REGULAR LOCAL RING
}

\author{
DANIEL KATZ AND VIJAY KODIYALAM
}

\begin{abstract}
Let $(R, m)$ be a two-dimensional regular local ring with infinite residue field. For a finitely generated, torsion-free $R$-module $A$, write $A_{n}$ for the $n$th symmetric power of $A$, mod torsion. We study the modules $A_{n}$, $n \geq 1$, when $A$ is complete (i.e., integrally closed). In particular, we show that $B \cdot A=A_{2}$, for any minimal reduction $B \subseteq A$ and that the $\operatorname{ring} \oplus_{n \geq 1} A_{n}$ is Cohen-Macaulay.
\end{abstract}

\section{INTRODUCTION}

The theory of complete ideals in two-dimensional regular local rings was initiated by Zariski to study linear systems on non-singular surfaces. In [Z] and in the appendix of [ZS], Zariski showed that the product of complete ideals is complete and that any complete ideal is uniquely expressible as a product of simple complete ideals. (Huneke's treatment $[\mathrm{H}]$ provides an excellent introduction to the subject.) These results have served as the springboard for further investigations into the properties of complete ideals. For example, Lipman has shown that Zariski's results hold for rational surface singularities (cf. [L]) and Cutkosky has characterized surface singularities for which products of complete ideals are complete and for which unique factorization holds (cf. [C1] and [C2]). In [HS] it is shown that complete ideals have many desirable analytic properties, e.g., their Rees algebras are Cohen-Macaulay.

Recently the second author extended part of Zariski's theory to torsion-free modules. Let $(R, m)$ be a two-dimensional regular local ring and suppose that $A$ and $B$ are finitely generated torsion-free $R$-modules. The main result of [Ko] states that if $A$ and $B$ are complete (see the definitions below), then so is their "product" $A \cdot B$, where $A \cdot B$ denotes the tensor product of $A$ and $B$ modulo its $R$-torsion. In this paper, we would like to continue in a like manner by studying symmetric powers, mod torsion, of complete modules. If $A$ is a finitely generated torsion-free $R$-module then $A$ embeds canonically into a free $R$-module $F$ (the "double dual" of $A$ ) so that the quotient has finite length. For each $n \geq 1$, let $F_{n}$ denote the $n$th symmetric power of $F$ and $A_{n}$ denote the image of the $n$th symmetric power of $A$ in $F_{n}$. Thus $A_{n}$ is the $n$th symmetric power of $A$, modulo its $R$ torsion. Let $I$ denote the ideal of maximal minors of the matrix whose columns generate $A$ and write $\lambda_{R}\left(F_{n} / A_{n}\right)$ for the length of $F_{n} / A_{n}$. The following theorem is the main result of this paper.

Received by the editors March 28, 1995.

1991 Mathematics Subject Classification. Primary 13B21, 13B22, 13H05, 13 H15.

The first author was partially supported by the General Research Fund at the University of Kansas.

(C)1997 American Mathematical Society 
Theorem 4.1. Let $(R, m)$ be a two-dimensional regular local ring and $A$ a finitely generated complete, torsion-free $R$-module. Set $r:=\operatorname{rank}(A)$. The following conditions hold:

(i) $B A=A_{2}$ for every minimal reduction $B \subseteq A$.

(ii) $I B=I A$ for every minimal reduction $B \subseteq A$.

(iii) The ring $\bigoplus_{n>0} A_{n}$ is Cohen-Macaulay.

(iv) The ring $\bigoplus_{n \geq 0} A_{n} / I A_{n}$ is Cohen-Macaulay.

(v) $\lambda_{R}\left(F_{n+1} / A_{n+1}\right)=\lambda_{R}(F / B) \cdot\left(\begin{array}{c}n+r+1 \\ r+1\end{array}\right)-\lambda_{R}(A / B) \cdot\left(\begin{array}{c}n+r \\ r\end{array}\right)$, for all $n \geq 0$ and every minimal reduction $B \subseteq A$.

In the statement of the theorem, $B A$ denotes the canonical image of $B \otimes_{R} A$ in $F_{2}$. Note that conditions (i) and (ii) are analogues of the reduction number one condition for complete ideals (cf. [LT]) and yield Briançon-Skoda like relations between $B$ and $A$. Parts (iii) and (iv) assert that the "Rees ring" and "associated graded ring" are Cohen-Macaulay while part (v) states that the Hilbert function associated to $A$ is a polynomial in $n$ for all values of $n$. The rank one case of these results was given in [HS]. The proof of the theorem will proceed in two steps. In section two we will prove that the reduction number one conditions hold for complete modules and in section three we show that parts (iii) and (v) follow from parts (i) and (ii). In section four we present the main theorem. In section one we establish our notation and definitions.

\section{Preliminaries}

Throughout, $(R, m)$ will denote a two-dimensional regular local ring with infinite residue field. Let $A$ be a non-free, finitely generated torsion-free $R$-module. Then $A$ has a projective resolution of the form

$$
0 \rightarrow G_{1} \rightarrow G_{0} \rightarrow A \rightarrow 0
$$

where $G_{0}$ and $G_{1}$ are finitely generated free $R$-modules. Dualizing this resolution yields

$$
0 \rightarrow A^{*} \rightarrow G_{0}^{*} \rightarrow G_{1}^{*} \rightarrow \operatorname{Ext}_{R}^{1}(A, R) \rightarrow 0,
$$

where "** denotes $R$-dual. Since $A$ is free on the punctured spectrum of $R$, $\operatorname{Ext}_{R}^{1}(A, R)$ has finite length. It follows that $A^{*}$ and therefore $A^{* *}$ are free $R$ modules. Since $A$ is torsion free, the canonical map $A \rightarrow A^{* *}$ is an embedding and because $A$ is free on the punctured spectrum of $R$, the cokernel of this embedding has finite length. Set $F:=A^{* *}$. It is not difficult to see that up to isomorphism, this is the only way that $A$ can be embedded in a free module such that the quotient has finite length. In other words, if $A \hookrightarrow F^{\prime}$ is an embedding of $A$ into the free module $F^{\prime}$ such that $\lambda_{R}\left(F^{\prime} / A\right)<\infty$, then there is an isomorphism from $F$ to $F^{\prime}$ taking the image of $A$ in $F$ onto the image of $A$ in $F^{\prime}$. So, we assume henceforth that $A \subseteq F$ and $\lambda_{R}(F / A)<\infty$. Set $r:=\operatorname{rank}(A)=\operatorname{rank}(F)$ and $m:=\mu(A)$, the minimal number of generators of $A$. If we fix a basis for $F$, we may identify the generators of $A$ with the columns of an $r \times m$ matrix. Write $C_{1}, \ldots, C_{m}$ for these column vectors and set $I:=I_{r}(A)$, the ideal of $r \times r$ minors of the corresponding matrix.

Now, let $F_{n}$ denote the $n$th symmetric power of $F$ and $A_{n}$ denote the image of the $n$th symmetric power of $A$ in $F_{n}$. Thus $\mathcal{F}:=\bigoplus_{n \geq 0} F_{n}$ is the symmetric algebra of $F$ and $\mathcal{A}:=\bigoplus_{n \geq 0} A_{n} \subseteq \mathcal{F}$ is the symmetric algebra of $A$, modulo its $R$-torsion. 
Note that $\operatorname{dim}(\mathcal{A})=\operatorname{dim}(\mathcal{F})=r+2$. In analogy with the case for ideals, we refer to $\mathcal{A}$ as the Rees ring of $R$ with respect to $A$. We shall frequently identify $A$ and $F$ with the degree one components of $\mathcal{A}$ and $\mathcal{F}$ and let the context determine when we mean $A$ or $A_{1}$ or $F$ or $F_{1}$. Additionally, for any $R$-module $B \subseteq F$, for any $f \in B_{m}$ and $g \in A_{n}$, we write $f g$ for the corresponding element of $F_{n+m}$. In other words, all sums and products we consider occur inside $\mathcal{F}$.

We now recall the notion of integral closure as it applies to modules. This definition was recently given by Rees in $[R]$, though its origin can be traced to the appendix of $[\mathrm{ZS}]$. The integral closure of $A$, denoted $A^{\prime}$, is defined to be the intersection of the modules $A V \cap F$ as $V$ ranges over the discrete valuation rings between $R$ and its quotient field $K$. By $A V$ we mean the $V$-submodule of $F \otimes_{R} K$ generated by $A$. Alternately, $A^{\prime}$ may be described as the degree one component of the integral closure of $\mathcal{A}$. $A$ is said to be integrally closed or complete if $A=A^{\prime}$. Closely related to the concept of the integral closure is the notion of reduction. An $R$-module $B \subseteq A$ is said to be a reduction of $A$ if $B^{\prime}=A^{\prime}$. Equivalently, $B$ is a reduction of $A$ if and only if $\mathcal{A}$ is a finitely generated module over $\mathcal{B}$, the Rees ring of $R$ with respect to $B$. Thus, by the Artin-Rees lemma, $B_{1} A_{n}=A_{n+1}$, for $n>>0$. A reduction $B \subseteq A$ is said to be a minimal reduction of $A$ if $B$ properly contains no further reductions of $A$. If $B \subseteq A$ is a minimal reduction, then the reduction number of $A$ with respect to $B$, denoted $\operatorname{rd}_{B}(A)$, is the smallest $n$ such that $A_{n+1}=B_{1} A_{n}$. $A$ is said to have reduction number one, if $B \neq A$ and $\operatorname{rd}_{B}(A)=1$, for some minimal reduction $B$. Just as for ideals, any minimal generating set for a minimal reduction of $A$ extends to a minimal generating set for $A$ itself. Moreover, it is not hard to see that $\lambda_{R}(F / B)<\infty$ for any reduction $B$. It follows from [BR, Thm. 3.1] that for $n>>0$, the functions $\lambda_{R}\left(F_{n} / B_{n}\right)$ and $\lambda_{R}\left(F_{n} / A_{n}\right)$ are polynomials in $n$ having degree $r+1$. The normalized leading coefficients of these polynomials are denoted $e(B)$ and $e(A)$, respectively, and are called the Buchsbaum-Rim multiplicities of $B$ and $A$. By [BR, Prop. 3.8], if $\mu(B)=r+1$, then $e(B)=\lambda_{R}(F / B)$. The following proposition records the basic facts we need concerning reductions.

Proposition 1.1. Let $A \subseteq F$ be as above. Then

(i) $e(B)=e(A)$, for any reduction $B \subseteq A$.

(ii) Any minimal reduction $B \subseteq A$ is minimally generated by $r+1$ elements. Consequently, if $B$ is a minimal reduction, then $e(A)=\lambda_{R}(F / B)$.

(iii) For any minimal reduction $B \subseteq A$, the Rees ring of $R$ with respect to $B$ is a hypersurface. In particular, the Rees ring equals the symmetric algebra for any minimal reduction $B$.

Proof. Let $B \subseteq A$ be a reduction. Then $\mathcal{A} / \mathcal{B}$ is a finitely generated $\mathcal{B}$-module whose graded components $A_{n} / B_{n}$ have finite length over $R$. Since the annihilator of $\mathcal{A} / \mathcal{B}$ contains a power of $m$, it follows that $\operatorname{dim}_{\mathcal{B}}(\mathcal{A} / \mathcal{B}) \leq r+1$. Thus the polynomial which for large $n$ gives $\lambda_{R}\left(A_{n} / B_{n}\right)$ has degree less than or equal to $r$. It follows immediately from this that $e(B)=e(A)$. That any minimal reduction $B \subseteq A$ is minimally generated by $r+1$ elements and that these elements extend to a minimal generating set for $A$ follow immediately from the Noether normalization lemma applied to $\mathcal{A} / m \mathcal{A}$ once we observe that $\operatorname{dim}(\mathcal{A} / m \mathcal{A})=r+1$, i.e., $\operatorname{height}(m \mathcal{A})=1$. Of course, height $(m \mathcal{A}) \leq 2$. Suppose that height $(m \mathcal{A})=2$. Then $\operatorname{dim}(\mathcal{A} / m \mathcal{A})=r$. By the normalization lemma, there exists a reduction $B \subseteq A$ with $\mu(B)=r$. Thus $B$ is a free $R$-module and therefore $\mathcal{B}$ is integrally closed. Therefore, $B=A$, and $A$ is free, a contradiction. Thus $\operatorname{dim}(\mathcal{A} / m \mathcal{A})=r+1$ and the first statement in 
part (ii) follows. The second statement follows from the remarks preceding the proposition.

To prove part (iii), let $B \subseteq A$ be a minimal reduction. By what we have just shown, we may assume that $B$ is generated by the first $r+1$ generators of $A$, i.e., the column vectors $C_{1}, \ldots, C_{r+1}$. Let $\tilde{B}$ denote the $r \times(r+1)$ matrix whose columns are the $C_{i}$ 's and for each $1 \leq i \leq r+1$, let $\Delta_{i}$ be $(-1)^{i+1}$ times the determinant obtained by deleting the $i$ th column of $\tilde{B}$. Thus

$$
\Delta_{1} C_{1}+\cdots+\Delta_{r+1} C_{r+1}=0 .
$$

Now, map $R\left[W_{1}, \ldots, W_{r+1}\right]$ onto $\mathcal{B}$ by sending $W_{i}$ to $C_{i}$ and denote by $L$ the kernel of this map. Clearly, $f:=\Delta_{1} W_{1}+\cdots+\Delta_{r+1} W_{r+1} \in L$. However, since $B_{P}=F_{P}$ for all non-maximal primes $P \subseteq R$, it follows that $f$ generates $L$ locally at each $P$. Thus for some $s \geq 1$, we have $m^{s} L \subseteq f \cdot R\left[W_{1}, \ldots, W_{r+1}\right]$. Since $R$ is regular, we conclude $L=f \cdot R\left[W_{1}, \ldots, W_{r+1}\right]$. This gives the desired conclusions.

\section{Reduction Number one}

In this section we show that complete, torsion-free $R$-modules have reduction number one. The idea is to induct on the Buchsbaum-Rim multiplicity and to use the facts that complete modules are contracted from quadratic transformations of $R$ and that the Buchsbaum-Rim multiplicity decreases upon passage to quadratic transforms. This strategy was introduced for ideals in $[\mathrm{H}]$ and $[\mathrm{HS}]$ and successfully adapted to modules in $[\mathrm{Ko}]$. We begin by describing in more detail the process of taking quadratic transforms of modules.

Let $K$ denote the quotient field of $R$. A (first) quadratic transformation of $R$ is a two-dimensional regular local ring $T$ between $R$ and $K$ obtained by localizing a ring of the form $R[m / x]$ at a height two maximal ideal, for some $x \in m \backslash m^{2}$. Let $A$ be a finitely generated torsion-free $R$-module, $F:=A^{* *}$ and $r:=\operatorname{rank}(A)$. We consider the module $A T$, i.e., the $T$-submodule of $F \otimes_{R} K$ generated by $A$ and call this the transform of $A$ over $T$. Note that if $A=I$ is an ideal, this differs from the usual notion of transform where the greatest common divisor in $T$ of the generators of $I$ has been factored out. We shall denote the standard transform by $I^{T}$. Thus $I^{T}$ is primary for the maximal ideal of $T$, while $I T$ is not. Of course, as $T$-modules, these ideals are isomorphic. For any birational extension $R \subseteq S$, we say that $A$ is contracted from $S$ if $A S \cap F=A$. Finally, we write $\operatorname{ord}_{R}(A)$ for the $m$-adic order of $A$, by which we mean $\operatorname{ord}_{R}\left(I_{r}(A)\right)$, the $m$-adic order of $I_{r}(A)$. The following theorem summarizes the results from $[\mathrm{Ko}]$ that we shall use in the proof of Proposition 2.2. In the statement of Theorem 2.1, a property is said to hold for sufficiently general $x \in m \backslash m^{2}$ if there exist ideals $I_{1}, \ldots, I_{h}$ properly contained in $m$ such that the property holds for all $x \notin m^{2} \cup I_{1} \cup \cdots \cup I_{h}$.

Theorem 2.1. Let $A$ be a finitely generated torsion-free $R$-module and set $I:=$ $I_{r}(A)$. The following statements hold.

(i) $\mu(A)=\operatorname{ord}_{R}(A)+r$ if and only if for sufficiently general $x \in m \backslash m^{2}, A$ is contracted from $S:=R[m / x]$.

(ii) If $A$ is complete, then for sufficiently general $x \in m \backslash m^{2}, A$ is contracted from $S:=R[m / x]$.

(iii) If $T$ is any quadratic transform, then $e(A T)<e(A), I_{r}(A T)=I^{T}$ and $A T$ is complete if $A$ is complete.

(iv) If $A$ is complete, then I, IA and $A_{2}$ are complete. 
Proposition 2.2. Let $A$ be a finitely generated complete, torsion-free $R$-module, $B \subseteq A$ a minimal reduction and $I:=I_{r}(A)$. Then

(i) $B A=A_{2}$.

(ii) $I B=I A$.

Proof. The proof in each case is similar and proceeds by induction on $e(A)$. If $e(A)=0$, then $A$ is free. Thus $B=A$ and there is nothing to prove. Suppose $e(A)>$ 0 . By Theorem 2.1, $A_{2}$ and $I A$ are complete. Suppose we could show that $\mu(B A)=$ $\operatorname{ord}_{R}(B A)+\operatorname{rank}(B A)$ and $\mu(I B)=\operatorname{ord}_{R}(I B)+\operatorname{rank}(I B)$. Then by Theorem 2.1 , there exists $x \in m \backslash m^{2}$ such that $A_{2}, B A, I A$ and $I B$ are contracted from $S:=R[m / x]$. For every transform $T$ associated to $S, e(A T)<e(A)$. Therefore $B A T=A_{2} T$ for all such transforms, so $B A S=A_{2} S$. Thus $B A=B A S \cap R=$ $A_{2} S \cap R=A_{2}$. Similarly, since $I_{r}(A T)=I_{r}(A)^{T}, I B T=I A T$ for all transforms $T$, so $I B S=I A S$. Thus $I B=I B S \cap R=I A S \cap R=I A$.

To prove $\mu(B A)=\operatorname{ord}_{R}(B A)+\operatorname{rank}(B A)$, note that $\operatorname{ord}_{R}(B A)=\operatorname{ord}_{R}\left(A_{2}\right)$, since $B A$ is a reduction of $A_{2}$. Since the ideal of maximal minors of $A_{2}$ is $I^{r+1}$, it follows that $\operatorname{ord}_{R}(B A)=(r+1) \operatorname{ord}_{R}(A)=(r+1)(m-r)$. $\operatorname{Thus~ord~}_{R}(B A)+$ $\operatorname{rank}(B A)=(r+1)(m-r)+\left(\begin{array}{c}r+1 \\ 2\end{array}\right)$. On the other hand, $B A=B_{2}+B L$, where $L$ is the submodule of $F$ generated by $C_{r+2}, \ldots, C_{m}$. Writing $\mathcal{B}$ for the Rees algebra of $B$, it follows from the proof of Proposition 1.1 that $\mathcal{B} / \mathrm{mB}$ is a polynomial ring in $r+1$ variables over $R / m$. Thus $\mu\left(B_{2}\right)=\left(\begin{array}{c}r+2 \\ 2\end{array}\right)$. It follows that $B A$ requires no more than $\left(\begin{array}{c}r+2 \\ 2\end{array}\right)+(r+1)(m-r-1)=\left(\begin{array}{c}r+1 \\ 2\end{array}\right)+(r+1)(m-r)$ generators. It therefore remains to see that the generators of $B_{2}+B L$ that we've accounted for are a minimal generating set. Now, suppose that we have an $R$ linear combination of these elements equal to zero. We need to show that each coefficient belongs to $m$. This can be seen as follows. We may rewrite the given relation as an $\mathcal{A}$ linear combination of $C_{1}, \ldots, C_{r+1}$, where the new coefficents have degree one. Suppose we could show that the $\mathcal{A}$ coefficents belong to $B_{1}$. Then, as the column vectors $C_{1}, \ldots, C_{m}$ minimally generate $A$, we are precluded from having any unit coefficents in the original relation on the generators of $B_{2}+B L$. That the degree one coefficients belong to $B_{1}$ is a consequence of the claim proven in Proposition 3.4 below (see $(\star))$. Thus $\mu(B A)=\operatorname{ord}_{R}(B A)+\operatorname{rank}(B A)$, as desired.

Similarly, to see $\mu(I B)=\operatorname{ord}_{R}(I B)+\operatorname{rank}(I B)$, note that

$$
\begin{aligned}
\operatorname{ord}_{R}(I B)+\operatorname{rank}(I B) & =\operatorname{ord}_{R}(I) \operatorname{rank}(B)+\operatorname{ord}_{R}(B)+\operatorname{rank}(B) \\
& =(m-r)(r+1)+r .
\end{aligned}
$$

On the other hand, since $A$ is complete, $I$ is complete. Thus

$$
\mu(I)=\operatorname{ord}_{R}(I)+1=m-r+1 .
$$

Let $\rho_{1}, \ldots, \rho_{m-r+1}$ minimally generate $I$. Then the $(m-r+1)(r+1)$ column vectors $\rho_{i} C_{j}$ generate $I B$, where $C_{1}, \ldots, C_{r+1}$ are the column vectors generating $B$. Write $J$ for the ideal of $r \times r$ minors associated to $B$. Since $B$ is a reduction of $A, J$ is a reduction of $I$. It follows that $J \nsubseteq m I$. Therefore, without loss of generality, we may assume $\rho_{1}=\Delta_{1}$ (in the notation of Proposition 1.1). Since $\Delta_{1} C_{1}+\cdots+\Delta_{r+1} C_{r+1}=0, \rho_{1} C_{1}$ is a redundant generator. If we show that the remaining column vectors $\rho_{i} C_{j}$ minimally generate $I B$, we will be done. Suppose 
that we have a relation $\sum_{i, j} \alpha_{i j} \rho_{i} C_{j}=0$, for some $\alpha_{i j} \in R$, with $\alpha_{11}=0$. Thus

$$
\left(\sum_{i} \alpha_{i 1} \rho_{i}\right) \cdot C_{1}+\cdots+\left(\sum_{i} \alpha_{i, r+1} \rho_{i}\right) \cdot C_{r+1}=0 .
$$

By the proof of Proposition 1.1, there exists $\gamma \in R$ such that $\sum_{i} \alpha_{i t} \rho_{i}=\gamma \Delta_{t}$, for all $1 \leq t \leq r+1$. Consider this equation for $t=1$. Since $\rho_{1}=\Delta_{1}$ and $\alpha_{11}=0$, if $\gamma \notin m$, then $\rho_{1}$ would belong to the ideal generated by $\rho_{2}, \ldots, \rho_{m-r+1}$, a contradiction. Thus $\gamma \in m$. It now follows easily that each $\alpha_{i j} \in m$, which is what we want.

Remark. Recall that the Briançon-Skoda theorem yields for any ideal $I \subseteq R$, $\left(I^{\prime}\right)^{2} \subseteq I$. This follows from the stronger result that $I^{\prime}$ has reduction number one. Proposition 2.2 gives similar Briançon-Skoda relations for modules. We record these as a corollary.

Corollary 2.3. Let $A \subseteq F$ be a finitely generated torsion-free $R$-module and $I:=$ $I_{r}(A)$. Then

(i) $\left(A^{\prime}\right)_{2} \subseteq F A$.

(ii) $I^{\prime} A^{\prime} \subseteq A$.

Proof. Let $B \subseteq A$ be a minimal reduction. Then $B$ is also a minimal reduction for the complete module $A^{\prime}$. Therefore the corollary follows immediately from the preceding proposition.

\section{Consequences of Reduction number one}

In this section we are going to use the reduction number one condition to see that the Rees algebra $\mathcal{A}$ is Cohen-Macaulay and to deduce that the Hilbert function $\lambda_{R}\left(F_{n+1} / A_{n+1}\right)$ is a polynomial in $n$ for all $n$. These consequences undoubtedly follow from the reduction number one condition in more general settings, but for now our techniques and exposition remain confined to the setting we have established.

Before moving on to Proposition 3.2, we will need to establish some notation. We assume that $A \subseteq F$ is minimally generated by $m$ column vectors. If $B \subseteq A$ is a minimal reduction, then $B$ is minimally generated by $r+1$ column vectors, which we take to be the first $r+1$ generators of $A$. We let $\tilde{B}$ denote the $r \times(r+1)$ matrix whose columns are the generators of $B$ and $\Delta_{i}$ be $(-1)^{i+1}$ times the determinant obtained by deleting the $i$ th column of $\tilde{B}$. For each $r+2 \leq j \leq m$, we let $H_{j}$ denote the $r \times(r+2)$ matrix obtained by adding the $j$ th column vector generating $A$ to the matrix $\tilde{B}$. For $1 \leq k \neq l \leq r+2, \Delta_{k, l}\left(H_{j}\right)$ will denote $(-1)^{k+l}$ times the determinant of the submatrix of $H_{j}$ obtained by deleting the $k$ th and $l$ th columns. There are numerous "generic relations" as well as the Plücker relations occurring among the minors of $A$ and its columns (when $m>r+1$ ). Fortunately, we need only a few. As before, we let $C_{1}, \ldots, C_{m}$ denote the column vectors generating $A$. We will express the relations we need in terms of the minors of the $H_{j}$ and the columns $C_{i}$. For $r+2 \leq j \leq m$ we have the two sets of relations

$$
\begin{aligned}
& (*) \quad-\Delta_{1,2}\left(H_{j}\right) C_{1}+\Delta_{2,3}\left(H_{j}\right) C_{3}+\cdots+\Delta_{2, r+1}\left(H_{j}\right) C_{r+1}+\Delta_{2, r+2}\left(H_{j}\right) C_{j}=0, \\
& (* *) \quad \Delta_{1,2}\left(H_{j}\right) C_{2}+\Delta_{1,3}\left(H_{j}\right) C_{3}+\cdots+\Delta_{1, r+1}\left(H_{j}\right) C_{r+1}+\Delta_{1, r+2}\left(H_{j}\right)\left(C_{j}\right)=0 .
\end{aligned}
$$


Note that for any $j$ and any $1 \leq k \leq r+1, \Delta_{k, r+2}\left(H_{j}\right)=(-1)^{r+1} \Delta_{k}$. Additionally, for each $r+2 \leq j \leq m$ and $3 \leq h \leq r+1$, we will need the Plücker relations

$(* * *) \quad \Delta_{1,2}\left(H_{j}\right) \Delta_{h, r+2}\left(H_{j}\right)-\Delta_{1, h}\left(H_{j}\right) \Delta_{2, r+2}\left(H_{j}\right)+\Delta_{1, r+2}\left(H_{j}\right) \Delta_{2, h}\left(H_{j}\right)=0$.

A good source for these relations is [BV]. We need a lemma before presenting Proposition 3.2 .

Lemma 3.1. Let $\tilde{B}$ be an $r \times(r+1)$ matrix with $I_{r}(\tilde{B}) m$-primary. Then after a possible application of elementary column operations, we may assume that $\Delta_{i}(\tilde{B})$ and $\Delta_{j}(\tilde{B})$ form a system of parameters for any pair $i \neq j$.

Proof. We begin with an observation. Let $Q_{1}, \ldots, Q_{h}$ be any collection of height one prime ideals. Then there exists a matrix $\widehat{B}$, obtained from $\tilde{B}$ by elementary column operations, such that $\Delta_{i}(\widehat{B}) \notin Q_{1} \cup \cdots \cup Q_{h}$ and $\Delta_{j}(\widehat{B})=\Delta_{j}(\tilde{B})$ for $j \neq i$. To see this, we may assume $i=1$. Let $J$ be the ideal generated by $\Delta_{2}(\tilde{B}), \ldots, \Delta_{r+1}(\tilde{B})$. Then $\left(\Delta_{1}(\tilde{B}), J\right) R \nsubseteq Q_{1} \cup \cdots \cup Q_{h}$. By the prime avoidance lemma $\left(\left[\right.\right.$ Kap, Thm. 256]), there exist $\rho_{2}, \ldots, \rho_{r+1}$ such that $\Delta_{1}(\tilde{B})+\rho_{2} \Delta_{2}(\tilde{B})+$ $\cdots+\rho_{r+1} \Delta_{r+1}(\tilde{B}) \notin Q_{i}$, for all $1 \leq i \leq h$. For each $2 \leq j \leq r+1$, we replace the $j$ th column $C_{j}$ of $\tilde{B}$ by $C_{j}+\rho_{j}^{\prime} C_{1}$, where $\rho_{j}^{\prime}=(-1)^{j} \rho_{j}$ and call the resulting matrix $\widehat{B}$. Then $\Delta_{1}(\widehat{B})=\Delta_{1}(\tilde{B})+\rho_{2} \Delta_{2}(\tilde{B})+\cdots+\rho_{r+1} \Delta_{r+1}(\tilde{B})$ and $\Delta_{j}(\widehat{B})=\Delta_{j}(\tilde{B})$, for $j \neq 1$, which is what we want. Now, if we let the collection of primes be the primes minimal over $\Delta_{1}(\tilde{B})$, we may apply the observation, as needed, to the remaining minors to assume that $\Delta_{1}(\tilde{B}), \Delta_{j}(\tilde{B})$ form a system of parameters for all $j \neq 1$. Now let the collection of primes be the union of the height one primes containing $\Delta_{1}(\tilde{B})$ or $\Delta_{2}(\tilde{B})$. Then we may again apply the observation to the remaining minors $\Delta_{3}(\tilde{B}), \ldots, \Delta_{r+1}(\tilde{B})$, as needed, to assume that $\Delta_{i}(\tilde{B}), \Delta_{j}(\tilde{B})$ form a system of parameters for $i=1,2$ and $j \neq i$. Continuing the process yields the result.

Proposition 3.2. Let $A$ be a finitely generated torsion-free $R$-module. Then $\mathcal{A}$ is Cohen-Macaulay if and only if the reduction number of $A$ with respect to $B$ is less than or equal to one for every minimal reduction $B \subseteq A$.

Proof. We retain the notation established before Lemma 3.1. Let $B \subseteq A$ be a minimal reduction. By Lemma 3.1 we may assume that $\Delta_{1}$ and $\Delta_{2}$ form a system of parameters in $R$. Set $\mathcal{J}:=\left(\Delta_{2}, C_{1}-\Delta_{1}, C_{2}, \ldots, C_{r+1}\right) \mathcal{A}$ and write $\mathcal{P}$ and $\mathcal{Q}$ respectively for the homogeneous maximal ideals of $\mathcal{A}$ and $\mathcal{B}$. Since $\mathcal{A}$ is a graded ring, to prove that $\mathcal{A}$ is Cohen-Macaulay, it is enough to show that $\mathcal{A}_{\mathcal{P}}$ is Cohen-Macaulay. We are going to derive the Cohen-Macaulay property of $\mathcal{A}_{\mathcal{P}}$ by comparing $\lambda_{R}(\mathcal{A} / \mathcal{J})$ and $e\left(\mathcal{J}_{\mathcal{P}}\right)$, the multiplicity of $\mathcal{J}_{\mathcal{P}}$. The proof will proceed in a number of steps.

We begin by showing that $\mathcal{J}$ is $\mathcal{P}$-primary. Now

$$
-C_{1}^{2}=-C_{1}\left(C_{1}-\Delta_{1}\right)+\Delta_{2} C_{2}+\cdots+\Delta_{r+1} C_{r+1}
$$

since $\Delta_{1} C_{1}+\cdots+\Delta_{r+1} C_{r+1}=0$. Hence $C_{1}^{2} \in \mathcal{J}$. Since $B$ is a reduction of $A$, it now follows that $\mathcal{A}_{+}^{s} \subseteq \mathcal{J}$, for an appropriate power $s$. On the other hand, $\Delta_{1}^{2} \in \mathcal{J}$, since $C_{1}^{2} \in \mathcal{J}$ and therefore $\left(\Delta_{1}^{2}, \Delta_{2}\right) \subseteq \mathcal{J}$. Since $\Delta_{1}, \Delta_{2}$ form a system of parameters it follows that some power of $m$ is contained in $\mathcal{J}$ and therefore that $\mathcal{J}$ is $\mathcal{P}$-primary. Since $\operatorname{dim}\left(\mathcal{A}_{\mathcal{P}}\right)=r+2, \mathcal{J}_{\mathcal{P}}$ is generated by a system of parameters in $\mathcal{A}_{\mathcal{P}}$. 
We now calculate $e\left(\mathcal{J}_{\mathcal{P}}\right)$. By the associativity formula, we may calculate the multiplicity of $\mathcal{J}_{\mathcal{Q}} \subseteq \mathcal{B}_{\mathcal{Q}}$. However, by Proposition 1.1, $\mathcal{B}$ is Cohen-Macaulay, therefore

$$
e\left(\mathcal{J}_{\mathcal{Q}}\right)=\lambda_{R}(\mathcal{B} / \mathcal{J B})
$$

Moreover, the proof of Proposition 1.1 shows that if we present $\mathcal{B}$ by mapping $R\left[W_{1}, \ldots, W_{r+1}\right]$ onto it in the obvious way, then the kernel of the resulting map is generated by the single linear polynomial $f:=\Delta_{1} W_{1}+\cdots+\Delta_{r+1} W_{r+1}$. Consequently,

$$
\begin{aligned}
\lambda_{R}(\mathcal{B} / \mathcal{J B}) & =\lambda_{R}\left(R\left[W_{1}, \ldots, W_{r+1}\right] /\left(\Delta_{2}, W_{1}-\Delta_{1}, W_{2}, \ldots, W_{r+1}, f\right)\right) \\
& =\lambda_{R}\left(R /\left(\Delta_{1}^{2}, \Delta_{2}\right) R\right) .
\end{aligned}
$$

It follows that $e\left(\mathcal{J}_{\mathcal{P}}\right)=\lambda_{R}\left(R /\left(\Delta_{1}^{2}, \Delta_{2}\right) R\right)=2 \lambda_{R}\left(R /\left(\Delta_{1}, \Delta_{2}\right) R\right.$ ) (since $R$ is CohenMacaulay).

Now, thinking of $\mathcal{A} / \mathcal{J}$ as a finite length $R$-module, we consider the submodule $L$ generated over $R$ by the elements $\left\{1^{\prime}, C_{r+2}^{\prime}, \ldots, C_{m}^{\prime}\right\}$, where $C_{j}^{\prime}$ denotes the image of $C_{j}$ in $\mathcal{A} / \mathcal{J}$. We are going to show that $\lambda_{R}(L)=2 \lambda_{R}\left(R /\left(\Delta_{1}, \Delta_{2}\right) R\right)$. Upon doing so, it will follow immediately that $\mathcal{A}$ is Cohen-Macaulay if and only if $\operatorname{rd}_{B}(A) \leq 1$. For $r+2 \leq i \leq m$, let $L_{i}$ denote the $R$ submodule of $L$ generated by $\left\{1^{\prime}, C_{r+2}^{\prime}, \ldots, C_{i}^{\prime}\right\}$. Then an easy induction argument shows that

$$
\lambda_{R}(L)=\lambda_{R}(R / \mathcal{J} \cap R)+\sum_{i=r+2}^{m-1} \lambda_{R}\left(R /\left(L_{i}:_{R} C_{i+1}^{\prime}\right)\right) .
$$

We now make two claims. The first claim is that $\mathcal{J} \cap R=\left(\Delta_{2}, \Delta_{1} J\right) R$, where

$$
J=\left(\Delta_{1}, \Delta_{2}, \Delta_{1,2}\left(H_{r+2}\right), \ldots, \Delta_{1,2}\left(H_{m}\right)\right) R .
$$

The second claim is that for $r+2 \leq i \leq m-1,\left(L_{i}:_{R} C_{i+1}^{\prime}\right)=\left(J_{i}: \Delta_{1,2}\left(H_{i+1}\right)\right)$, where $J_{i}=\left(\Delta_{1}, \Delta_{2}, \Delta_{1,2}\left(H_{r+2}\right), \ldots, \Delta_{1,2}\left(H_{i}\right)\right) R$. Suppose for the moment that these claims hold. Then

$$
\begin{aligned}
\lambda_{R}(L)= & \lambda_{R}(R / \mathcal{J} \cap R)+\sum_{i=r+2}^{m-1} \lambda_{R}\left(R /\left(L_{i}:_{R} C_{i+1}^{\prime}\right)\right) \\
= & \lambda_{R}\left(R /\left(\Delta_{2}, \Delta_{1} J\right)\right)+\sum_{i=r+2}^{m-1} \lambda_{R}\left(R /\left(J_{i}: \Delta_{1,2}\left(H_{i+1}\right)\right)\right) \\
= & \lambda_{R}\left(R /\left(\Delta_{1}, \Delta_{2}\right) R\right)+\lambda_{R}\left(\left(\Delta_{1}, \Delta_{2}\right) R /\left(\Delta_{1} J, \Delta_{2}\right)\right) \\
& +\sum_{i=r+2}^{m-1} \lambda_{R}\left(R /\left(J_{i}: \Delta_{1,2}\left(H_{i+1}\right)\right)\right) \\
= & \lambda_{R}\left(R /\left(\Delta_{1}, \Delta_{2}\right) R\right)+\lambda_{R}\left(\Delta_{1} R /\left(\Delta_{1} J, \Delta_{1} \Delta_{2}\right)\right) \\
& +\sum_{i=r+2}^{m-1} \lambda_{R}\left(R /\left(J_{i}: \Delta_{1,2}\left(H_{i+1}\right)\right)\right) \\
= & \lambda_{R}\left(R /\left(\Delta_{1}, \Delta_{2}\right) R\right)+\lambda_{R}(R / J)+\sum_{i=r+2}^{m-1} \lambda_{R}\left(R /\left(J_{i}: \Delta_{1,2}\left(H_{i+1}\right)\right)\right) .
\end{aligned}
$$


However, an easy induction shows that

$$
\lambda_{R}(R / J)+\sum_{i=r+2}^{m-1} \lambda_{R}\left(R /\left(J_{i}: \Delta_{1,2}\left(H_{i+1}\right)\right)\right)=\lambda_{R}\left(R /\left(\Delta_{1}, \Delta_{2}\right) R\right) .
$$

Thus, $\lambda_{R}(L)=2 \lambda_{R}\left(R /\left(\Delta_{1}, \Delta_{2}\right) R\right)$, as desired. It therefore remains to verify both claims.

To verify the first claim, suppose that $s \in \mathcal{J} \cap R$. Then we have an equation

$$
s=u \Delta_{2}+v_{1}\left(C_{1}-\Delta_{1}\right)+\sum_{j=2}^{r+1} v_{j} C_{j}
$$

with coefficients from $\mathcal{A}$. Comparing the degree zero and degree one terms in this equation gives rise to the equations

$$
\begin{gathered}
s=u_{0} \Delta_{2}-v_{01} \Delta_{1}, \\
0=u_{1} \Delta_{2}-v_{11} \Delta_{1}+\sum_{j=1}^{r+1} v_{0 j} C_{j},
\end{gathered}
$$

where $u_{0}$ and the $v_{0 j}$ are the degree zero components of $u$ and the $v_{j}$, and $u_{1}$ and the $v_{1 j}$ are the degree one components. We need to see that $v_{01} \in J$. Writing $u_{1}=\sum_{j=1}^{m} \alpha_{j} C_{j}, \alpha_{j} \in R$ and using $(*)$ (bearing in mind that $\Delta_{2}= \pm \Delta_{2, r+2}\left(H_{j}\right)$ for all $j$ ), we obtain

$$
u_{1} \Delta_{2}=\left(\Delta_{2} \alpha_{1}+\sum_{j=r+2}^{m} \pm \alpha_{j} \Delta_{1,2}\left(H_{j}\right)\right) C_{1}+\sum_{i=2}^{r+1} \gamma_{i} C_{i}
$$

for appropriate elements $\gamma_{i} \in R$ determined by (*). Writing $-v_{11}=\sum_{j=1}^{m} \beta_{j} C_{j}$, we may do likewise for $-v_{11} \Delta_{1}$, using $(* *)$. However, since we are only interested in the resulting coefficient of $C_{1}$, we simply write

$$
-v_{11} \Delta_{1}=\Delta_{1} \beta_{1} C_{1}+\sum_{i=2}^{r+1} \delta_{i} C_{i},
$$

for appropriate $\delta_{i} \in R$. We therefore obtain

$$
\begin{aligned}
0 & =u_{1} \Delta_{2}-v_{11} \Delta_{1}+\sum_{j=1}^{r+1} v_{0 j} C_{j} \\
& =\left(\Delta_{2} \alpha_{1}+\sum_{j=r+2}^{m} \pm \alpha_{j} \Delta_{1,2}\left(H_{j}\right)+\Delta_{1} \beta_{1}+v_{01}\right) C_{1}+\sum_{i=2}^{r+1} \mu_{i} C_{i},
\end{aligned}
$$

for appropriate $\mu_{i} \in R$. Since $\sum_{k=1}^{r+1} \Delta_{k} C_{k}=0$ is the only relation on $C_{1}, \ldots, C_{r+1}$ (by Proposition 1.1), it follows that

$$
\Delta_{2} \alpha_{1}+\sum_{j=r+2}^{m} \pm \alpha_{j} \Delta_{1,2}\left(H_{j}\right)+\Delta_{1} \beta_{1}+v_{01} \in \Delta_{1} R .
$$

In other words, $v_{01} \in J$, as desired. Conversely, suppose that

$$
s \in\left(\Delta_{2}, \Delta_{1} J\right)=\left(\Delta_{2}, \Delta_{1}^{2}, \Delta_{1} \Delta_{1,2}\left(H_{r+2}\right), \ldots, \Delta_{1} \Delta_{1,2}\left(H_{m}\right)\right) .
$$


Then there exist $t, s_{i} \in R$ such that

$$
s=t \Delta_{2}+s_{1} \Delta_{1}^{2}+\sum_{i=r+2}^{m} s_{i} \Delta_{1} \Delta_{1,2}\left(H_{i}\right) .
$$

Therefore

$$
\begin{aligned}
s= & t \Delta_{2}+s_{1}\left(\Delta_{1}\left(-C_{1}+\Delta_{1}\right)-\sum_{j=2}^{r+1} \Delta_{j} C_{j}\right)+\left(-C_{1}+\Delta_{1}\right) \sum_{i=r+2}^{m} s_{i} \Delta_{1,2}\left(H_{i}\right) \\
& +\sum_{i=r+2}^{m} s_{i} \Delta_{1,2}\left(H_{i}\right) C_{1} .
\end{aligned}
$$

However, $(*)$ implies that $\Delta_{1,2}\left(H_{i}\right) C_{1} \in\left(\Delta_{2}, C_{3}, \ldots, C_{r+1}\right) R$, for all $r+2 \leq i \leq m$. Thus, $s \in \mathcal{J} \cap R$ and we have verified the first claim.

We now proceed to verify the second claim. Suppose $s \in\left(L_{i}:_{R} C_{i+1}\right)$. Then a moment's reflection will show that we may write an equation

$$
s C_{i+1}=v_{1} \Delta_{1}+v_{2} \Delta_{2}+\sum_{j=1}^{r+1} u_{j}^{\prime} C_{j}+\sum_{j=r+2}^{i} t_{j} C_{j},
$$

where $v_{1}, v_{2} \in \mathcal{A}_{1}$ and $u_{j}^{\prime}, t_{j} \in R$. Equations $(*)$ and $(* *)$ show that $v_{1} \Delta_{1}$ and $v_{2} \Delta_{2}$ can be expressed as $R$ linear combinations of $C_{1}, \ldots, C_{r+1}$. Thus, we may rewrite the equation as

$$
s C_{i+1}=\sum_{j=1}^{r+1} u_{j} C_{j}+\sum_{j=r+2}^{i} t_{j} C_{j},
$$

for $u_{j} \in R$. Multiplying by $\Delta_{2}^{\prime}:=(-1)^{r+1} \Delta_{2}$ (and bearing in mind that $\Delta_{2}^{\prime}=$ $\Delta_{2, r+2}\left(H_{j}\right)$ for all $\left.j\right)$, we obtain

$$
s \Delta_{2}^{\prime} C_{i+1}=\sum_{j=1}^{r+1} u_{j} \Delta_{2}^{\prime} C_{j}+\sum_{j=r+2}^{i} t_{j} \Delta_{2, r+2}\left(H_{j}\right) C_{j} .
$$

Using $(*)$, we may rewrite this last equation as

$$
\begin{aligned}
s\left(\Delta_{1,2}\left(H_{i+1}\right) C_{1}-\sum_{k=3}^{r+1} \Delta_{2, k}\left(H_{i+1}\right) C_{k}\right) & \\
= & \sum_{j=1}^{r+1} u_{j} \Delta_{2}^{\prime} C_{j}+\sum_{j=r+2}^{i} t_{j}\left(-\Delta_{1,2}\left(H_{j}\right) C_{1}-\sum_{k=3}^{r+1} \Delta_{2, k}\left(H_{j}\right) C_{k}\right) .
\end{aligned}
$$

Thus

$$
\left(s \Delta_{1,2}\left(H_{i+1}\right)-u_{1} \Delta_{2}^{\prime}+\sum_{j=r+2}^{i} t_{j} \Delta_{1,2}\left(H_{j}\right)\right) C_{1}+\sum_{k=2}^{r+1} \alpha_{k} C_{k}=0,
$$

for appropriate $\alpha_{k} \in R$. As before, this implies that

$$
s \Delta_{1,2}\left(H_{i+1}\right)+u_{1} \Delta_{2}^{\prime}-\sum_{j=r+2}^{i} t_{j} \Delta_{1,2}\left(H_{j}\right) \in \Delta_{1} R .
$$


Thus $s \in\left(J_{i}: \Delta_{1,2}\left(H_{i+1}\right)\right)$, as desired. Finally, suppose that $s \in\left(J_{i}: \Delta_{1,2}\left(H_{i+1}\right)\right)$. For each $1 \leq h \leq r+1$, set $\Delta_{h}^{\prime}:=(-1)^{r+1} \Delta_{h}=\Delta_{h, r+2}\left(H_{j}\right)$. Then we have an equation

$$
s \Delta_{1,2}\left(H_{i+1}\right)=u \Delta_{1}^{\prime}+v \Delta_{2}^{\prime}+\sum_{k=r+2}^{i} t_{k} \Delta_{1,2}\left(H_{k}\right)
$$

For each $3 \leq h \leq r+1$, multiply this equation by $\Delta_{h}^{\prime}$. We get

$$
s \Delta_{1,2}\left(H_{i+1}\right) \Delta_{h}^{\prime}=u \Delta_{1}^{\prime} \Delta_{h}^{\prime}+v \Delta_{2}^{\prime} \Delta_{h}^{\prime}+\sum_{k=r+2}^{i} t_{k} \Delta_{1,2}\left(H_{k}\right) \Delta_{h}^{\prime} .
$$

Using the Plücker relations $(* * *)$ we obtain

$$
\begin{aligned}
& s\left(\Delta_{1, h}\left(H_{i+1}\right) \Delta_{2}^{\prime}-\Delta_{1}^{\prime} \Delta_{2, h}\left(H_{i+1}\right)\right) \\
& \quad=u \Delta_{1}^{\prime} \Delta_{h}^{\prime}+v \Delta_{2}^{\prime} \Delta_{h}^{\prime}+\sum_{k=r+2}^{i} t_{k}\left(\Delta_{1, h}\left(H_{k}\right) \Delta_{2}^{\prime}-\Delta_{1}^{\prime} \Delta_{2, h}\left(H_{k}\right)\right) .
\end{aligned}
$$

Therefore

$$
\begin{aligned}
\left(u \Delta_{h}^{\prime}\right. & \left.-\sum_{k=r+2}^{i} t_{k} \Delta_{2, h}\left(H_{k}\right)+s \Delta_{2, h}\left(H_{i+1}\right)\right) \Delta_{1}^{\prime} \\
& +\left(v \Delta_{h}^{\prime}+\sum_{k=r+2}^{i} t_{k} \Delta_{1, h}\left(H_{k}\right)-s \Delta_{1, h}\left(H_{i+1}\right)\right) \Delta_{2}^{\prime}=0 .
\end{aligned}
$$

Since $\Delta_{1}^{\prime}, \Delta_{2}^{\prime}$ form a regular sequence, we have that

$$
v \Delta_{h}^{\prime}+\sum_{k=r+2}^{i} t_{k} \Delta_{1, h}\left(H_{k}\right)-s \Delta_{1, h}\left(H_{i+1}\right)=-\mu_{h} \Delta_{1}^{\prime}
$$

for some $\mu_{h} \in R, 3 \leq h \leq r+1$. If we set $\mu_{2}:=u$ and bear in mind our original equation, we have that

$$
s \Delta_{1, h}\left(H_{i+1}\right)=\mu_{h} \Delta_{1}^{\prime}+v \Delta_{h}^{\prime}+\sum_{k=r+2}^{i} t_{k} \Delta_{1, h}\left(H_{k}\right)
$$

for $h=2, \ldots, r+1$. Thus

$$
\sum_{h=2}^{r+1} s \Delta_{1, h}\left(H_{i+1}\right) C_{h}=\sum_{h=2}^{r+1} \Delta_{1}^{\prime} \mu_{h} C_{h}+\sum_{h=2}^{r+1} v \Delta_{h}^{\prime} C_{h}+\sum_{k=r+2}^{i} t_{k}\left(\sum_{h=2}^{r+1} \Delta_{1, h}\left(H_{k}\right) C_{h}\right) .
$$

It follows from $(* *)$ that

$$
-s \Delta_{1}^{\prime} C_{i+1}=\Delta_{1}^{\prime} \sum_{h=2}^{r+1} \mu_{h} C_{h}+v\left(-\Delta_{1}^{\prime} C_{1}\right)+\sum_{k=r+2}^{i} t_{k}\left(-\Delta_{1}^{\prime} C_{k}\right) .
$$

Therefore, $s C_{i+1}$ is an $R$ linear combination of $C_{1}, \ldots, C_{i}$. It follows easily from this that $s C_{i+1}^{\prime} \in L_{i}$, as desired. Thus the second claim has been verified, and the proof of the proposition is now complete.

Remark. The proof of Proposition 3.2 shows that if $\operatorname{rd}_{B}(A)=1$, for some minimal reduction $B \subseteq A$, then $\operatorname{rd}_{B}(A)=1$, for every minimal reduction $B$. 
We are now going to turn our attention to showing that if $A$ has reduction number one, then the function $\lambda_{R}\left(F_{n+1} / A_{n+1}\right)$ is a polynomial in $n$ for all $n \geq 0$. We will first focus on a minimal reduction of $A$. To do this we need a definition. Let $B$ be any torsion-free $R$-module. $B$ is said to be a parameter module if $\mu(B)=$ $\operatorname{rank}(B)+1$. If we embed $B$ into a free module so that the quotient has finite length, then the matrix whose columns correspond to the generators of $B$ is a parameter matrix in the sense of $[\mathrm{BR}]$.

Proposition 3.3. Let $B$ be a finitely generated torsion-free parameter module, set $F:=B^{* *}$ and assume $\operatorname{rank}(B)=r$. Then for all $n \geq 0, \lambda_{R}\left(F_{n+1} / B_{n+1}\right)=$ $\lambda_{R}(F / B) \cdot\left(\begin{array}{c}n+r+1 \\ r+1\end{array}\right)$.

Proof. We induct on $n$ and $r$. If $r=1$, then $B$ is a parameter ideal and the result is well known. If $n=0$, the conclusion clearly holds. Suppose now that $r>1$ and $n>0$. Choosing a basis for $F$, we may regard the generators of $B$ as column vectors. Since $B$ is free on the punctured spectrum of $R$, basic element theory allows us to find a minimal generator $x \in B$, such that the ideal in $R$ generated by the entries of $x$ has height greater than or equal to 2 . Set $F^{\prime}:=F / R x$ and $B^{\prime}:=B / R x$. Then $B^{\prime} \subseteq F^{\prime}$ are torsion free $R$-modules. Of course, if $x$ is also a minimal generator for $F$, then $F^{\prime}$ is a free $R$-module. In any case, since $\lambda_{R}\left(F^{\prime} / B^{\prime}\right)<\infty,\left(F^{\prime}\right)^{*}=\left(B^{\prime}\right)^{*}$ is free of rank $r-1$. Thus $F^{\prime}$ and $B^{\prime}$ have the same double dual, say $G$. Note in the case that $R x$ is a summand of $F, F^{\prime}=G$. If $R x$ is not a summand of $F$, then $\mu\left(F^{\prime}\right)=r=\operatorname{rank}\left(F^{\prime}\right)+1$, so $F^{\prime}$ is a parameter module. Clearly $B^{\prime}$ is a parameter module. Thus, by induction on $r$,

$$
\lambda_{R}\left(G_{n+1} / F_{n+1}^{\prime}\right)=\lambda_{R}\left(G / F^{\prime}\right) \cdot\left(\begin{array}{c}
n+r \\
r
\end{array}\right)
$$

and

$$
\lambda_{R}\left(G_{n+1} / B_{n+1}^{\prime}\right)=\lambda_{R}\left(G / B^{\prime}\right) \cdot\left(\begin{array}{c}
n+r \\
r
\end{array}\right)
$$

Therefore,

$$
\lambda_{R}\left(F_{n+1}^{\prime} / B_{n+1}^{\prime}\right)=\lambda_{R}\left(F^{\prime} / B^{\prime}\right) \cdot\left(\begin{array}{c}
n+r \\
r
\end{array}\right)=\lambda_{R}(F / B) \cdot\left(\begin{array}{c}
n+r \\
r
\end{array}\right) .
$$

On the other hand, $\mathcal{F} / x \mathcal{F}$ and $\mathcal{B} / x \mathcal{B}$ are the symmetric algebras of $F^{\prime}$ and $B^{\prime}$. Since $F^{\prime}$ and $B^{\prime}$ are either free or parameter modules, their Rees algebras equal their symmetric algebras (Proposition 1.1). Thus we have exact sequences

$$
\begin{gathered}
0 \rightarrow F_{n} \stackrel{\cdot x}{\longrightarrow} F_{n+1} \rightarrow F_{n+1}^{\prime} \rightarrow 0 \\
0 \rightarrow B_{n} \stackrel{\cdot x}{\longrightarrow} B_{n+1} \rightarrow B_{n+1}^{\prime} \rightarrow 0
\end{gathered}
$$

from which it follows that the sequence

$$
0 \rightarrow F_{n} / B_{n} \stackrel{\cdot x}{\longrightarrow} F_{n+1} / B_{n+1} \rightarrow F_{n+1}^{\prime} / B_{n+1}^{\prime} \rightarrow 0
$$

is exact. Therefore,

$$
\lambda_{R}\left(F_{n+1} / B_{n+1}\right)=\lambda_{R}\left(F_{n} / B_{n}\right)+\lambda_{R}\left(F_{n+1}^{\prime} / B_{n+1}^{\prime}\right) .
$$


By induction on $n, \lambda_{R}\left(F_{n} / B_{n}\right)=\lambda_{R}(F / B) \cdot\left(\begin{array}{l}n+r \\ r+1\end{array}\right)$. Thus,

$$
\begin{aligned}
\lambda_{R}\left(F_{n+1} / B_{n+1}\right) & =\lambda_{R}(F / B) \cdot\left(\begin{array}{c}
n+r \\
r+1
\end{array}\right)+\lambda_{R}(F / B) \cdot\left(\begin{array}{c}
n+r \\
r
\end{array}\right) \\
& =\lambda_{R}(F / B) \cdot\left(\begin{array}{c}
n+r+1 \\
r+1
\end{array}\right),
\end{aligned}
$$

as desired.

Proposition 3.4. Let $A$ be a finitely generated torsion-free $R$-module and assume that the reduction number of $A$ is one. Then for all $n \geq 0, \lambda_{R}\left(F_{n+1} / A_{n+1}\right)=$ $e(A) \cdot\left(\begin{array}{c}n+r+1 \\ r+1\end{array}\right)-\lambda_{R}(A / B) \cdot\left(\begin{array}{c}n+r \\ r\end{array}\right)$, for any minimal reduction $B \subseteq A$.

Proof. Let $B \subseteq A$ be a minimal reduction. By the remark above, since the reduction number of $A$ is one, $A_{n+1}=A_{1} B_{n}$, for all $n \geq 0$. Consider the exact sequences

$$
\begin{aligned}
& 0 \rightarrow F_{1} B_{n} / A_{1} B_{n} \rightarrow F_{n+1} / A_{n+1} \rightarrow F_{n+1} / F_{1} B_{n} \rightarrow 0 \\
& 0 \rightarrow F_{1} B_{n} / B_{n+1} \rightarrow F_{n+1} / B_{n+1} \rightarrow F_{n+1} / F_{1} B_{n} \rightarrow 0 .
\end{aligned}
$$

It follows that

$$
\lambda_{R}\left(F_{n+1} / A_{n+1}\right)=\lambda_{R}\left(F_{n+1} / B_{n+1}\right)+\lambda_{R}\left(F_{1} B_{n} / A_{1} B_{n}\right)-\lambda_{R}\left(F_{1} B_{n} / B_{n+1}\right) .
$$

We now make the following claim. Let $C_{1}, \ldots, C_{r+1}$ denote the column vectors generating $B$ and set $N:=\left(\begin{array}{c}n+r \\ r\end{array}\right)$. If $f_{1}, \ldots, f_{N}$ are linear forms in $\mathcal{F}$ satisfying

$$
f_{1} C_{1}^{n}+f_{2} C_{1}^{n-1} C_{2}+\cdots+f_{N-1} C_{r} C_{r+1}^{n-1}+f_{N} C_{r+1}^{n}=0,
$$

then each $f_{i} \in B$. Suppose the claim holds. Let $\phi: F^{N} \rightarrow F_{1} B_{n}$ be the surjective $R$-module homorphism taking the $N$-tuple $\left(f_{1}, \ldots, f_{N}\right)$ to $f_{1} C_{1}^{n}+\cdots+f_{N} C_{r+1}^{n}$. It follows from the claim that the induced maps

$$
\phi_{1}:(F / A)^{N} \rightarrow F_{1} B_{n} / A_{1} B_{n} \quad \text { and } \quad \phi_{2}:(F / B)^{N} \rightarrow F_{1} B_{n} / B_{n+1}
$$

are isomorphisms. Thus

$$
\lambda_{R}\left(F_{1} B_{n} / A_{1} B_{n}\right)=\lambda_{R}(F / A) \cdot\left(\begin{array}{c}
n+r \\
r
\end{array}\right)
$$

and

$$
\lambda_{R}\left(F_{1} B_{n} / B_{n+1}\right)=\lambda_{R}(F / B) \cdot\left(\begin{array}{c}
n+r \\
r
\end{array}\right) .
$$

Since $\lambda_{R}\left(F_{n+1} / B_{n+1}\right)=e(A) \cdot\left(\begin{array}{c}n+r+1 \\ r+1\end{array}\right)$, the proposition follows, once we verify the claim.

To verify the claim, we consider it to be a statement concerning parameter modules and prove the statement by induction on $r$. If $r=1$, the result is wellknown. Suppose that $r>1$. It follows from Lemma 3.1, that if we regard the generators of $B$ as column vectors in $F$, then we may assume that for each $C_{i}$, $1 \leq i \leq r+1$, the ideal in $R$ generated by the entries of $C_{i}$ has height greater than or equal to 2. Set $x:=C_{i}, B^{\prime}:=B / R x$ and $F^{\prime}:=F / R x$. As in the proof of Proposition 3.3, $B$ and $F$ have the same double dual $G, \mathcal{B} / x \mathcal{B}$ equals the Rees ring of $R$ with respect to $B^{\prime}$ and $\mathcal{F} / x \mathcal{F}$ equals the Rees ring of $R$ with respect to $F^{\prime}$. Thus $\mathcal{B} / x \mathcal{B} \subseteq \mathcal{F} / x \mathcal{F} \subseteq \mathcal{G}$, the symmetric algebra of $G$. If we now think of $(\star)$ as an equation in $\mathcal{F}$, we may reduce the equation $\bmod x \mathcal{F}$, where it becomes an equation in $\mathcal{G}$. By induction on $r$ we conclude that every coefficient of a term in 
$(\star)$ not involving $C_{i}$ belongs to $B$. Varying $i$ will cover all coefficients in $(\star)$, i.e., each $f_{i} \in B$, as desired.

\section{THE MAIN THEOREM}

We are now ready for the main result of the paper.

Theorem 4.1. Let $(R, m)$ be a two-dimensional regular local ring and $A$ a finitely generated complete, torsion-free $R$-module. The following conditions hold:

(i) $B A=A_{2}$ for every minimal reduction $B \subseteq A$.

(ii) $I B=I A$ for every minimal reduction $B \subseteq A$.

(iii) The Rees ring $\mathcal{A}$ is Cohen-Macaulay.

(iv) The ring $\mathcal{A} / I \mathcal{A}$ is Cohen-Macaulay.

(v) $\lambda_{R}\left(F_{n+1} / A_{n+1}\right)=e(A) \cdot\left(\begin{array}{c}n+r+1 \\ r+1\end{array}\right)-\lambda_{R}(A / B) \cdot\left(\begin{array}{c}n+r \\ r\end{array}\right)$, for all $n \geq 0$ and every minimal reduction $B \subseteq A$.

Proof. Parts (i) and (ii) follow from Proposition 2.2. Parts (iii) and (iv), follow from part (i) via Propostions 3.2 and 3.4. To see that $\mathcal{A} / I \mathcal{A}$ is Cohen-Macaulay, we retain the notation of Proposition 3.2. Since the images of $C_{1}, \ldots, C_{r+1}$ form a homogeneous system of parameters in $\mathcal{A} / I \mathcal{A}$, it remains to see that they also form a regular sequence. For this, suppose we have a relation

$$
f_{1} C_{1}+\cdots+f_{r+1} C_{r+1} \in I \mathcal{A},
$$

where each $f_{i} \in \mathcal{A}$ is homogeneous, say of degree $n$. If $n=0$, then, since $I A=I B$, we may write an equation

$$
f_{1} C_{1}+\cdots+f_{r+1} C_{r+1}=s_{1} C_{1}+\cdots+s_{r+1} C_{r+1},
$$

with each $s_{i} \in I$. Since $\Delta_{1} C_{1}+\cdots+\Delta_{r+1} C_{r+1}=0$ is the only relation on the $C_{i}$ 's (over $R$ ), it follows that each $f_{i} \in I$, which is what we want. Suppose now that $n \geq 1$. Then $f_{1} C_{1}+\cdots+f_{r+1} C_{r+1} \in I A_{n+1}=I A_{n} B$. Thus there exist $g_{i} \in I A_{n}$ such that

$$
f_{1} C_{1}+\cdots+f_{r+1} C_{r+1}=g_{1} C_{1}+\cdots+g_{r+1} C_{r+1} .
$$

Setting $f_{i}^{\prime}=f_{i}-g_{i}$, we obtain

$$
f_{1}^{\prime} C_{1}+\cdots+f_{r+1}^{\prime} C_{r+1}=0
$$

Thus,

$$
f_{1}^{\prime} \Delta_{1}+f_{1}^{\prime}\left(C_{1}-\Delta_{1}\right)+f_{2}^{\prime} C_{2}+\cdots+f_{r+1}^{\prime} C_{r+1}=0 .
$$

However, the generic relations $(* *)$ imply that $\Delta_{1} A$ is contained in the submodule $I C_{2}+\cdots+I C_{r+1}$. Thus we may rewrite this last equation as

$$
f_{1}^{\prime}\left(C_{1}-\Delta_{1}\right)+h_{2} C_{2}+\cdots+h_{r+1} C_{r+1}=0,
$$

where $h_{i}=f_{i}^{\prime}-k_{i}$, for some $k_{i} \in I A_{n-1}$. Since $C_{1}-\Delta_{1}, C_{2}, \ldots, C_{r+1}$ form a regular sequence in $\mathcal{A}_{\mathcal{P}}$, the coefficients of this last equation give rise to a column vector belonging to the Koszul relations on $C_{1}-\Delta_{1}, C_{2}, \ldots, C_{r+1}$. Interpreting this modulo $I \mathcal{A}$ yields the result.

Remark. (a) When $A=I$ is an ideal, not necessarily complete, it is known that the conditions in Theorem 4.1 are equivalent. We conjecture that the conditions in the theorem are equivalent for modules which are not necessarily complete. Our proof almost shows this; however, we have not been able to demonstrate the equivalence 
of conditions (i) and (ii) for an arbitrary module $A$. Assuming this, then the conditions are readily seen to be equivalent. We record this as a corollary.

(b) In [HS] it is shown that if $I \subseteq R$ is a complete ideal, then the Rees ring of $R$ with respect to $I$ localized at its homogeneous maximal ideal is Cohen-Macaulay with minimal multiplicity. This continues to hold for complete modules, as we show in the corollary below.

Corollary 4.2. Let $A$ be a finitely generated torsion-free R-module.

(i) Conditions (i), (iii), and (iv) in Theorem 4.1 are equivalent. If conditions (i) and (ii) are equivalent, then conditions (i)-(v) are equivalent.

(ii) Let $\mathcal{P}$ denote the homogeneous maximal ideal of $\mathcal{A}$. Then $\mathcal{A}_{\mathcal{P}}$ is CohenMacaulay with minimal multiplicity if and only if $A$ has reduction number less than or equal to 1 and $\mu(A)=\operatorname{ord}_{R}(A)+\operatorname{rank}(A)$. In particular, if $A$ is complete, then $\mathcal{A}_{\mathcal{P}}$ is Cohen-Macaulay with minimal multiplicity.

Proof. The equivalence of (i) and (iii) follows from Proposition 3.2. That (i) implies (v) is Proposition 3.4. Conversely, suppose condition (v) holds. Then

$$
\lambda_{R}\left(F_{2} / A_{2}\right)=\lambda_{R}(F / B)+(r+1) \lambda_{R}(F / A) .
$$

From the proof of Proposition 3.4, we have

$$
(r+1) \lambda_{R}(F / A)=\lambda_{R}\left(F_{1} B_{1} / A_{1} B_{1}\right) \quad \text { and } \quad \lambda_{R}\left(F_{1} B_{1} / B_{2}\right)=(r+1) \lambda_{R}(F / B) .
$$

By Proposition 3.3, $\lambda_{R}\left(F_{2} / B_{2}\right)=(r+2) \lambda_{R}(F / B)$. Thus

$$
\begin{aligned}
\lambda_{R}\left(F_{2} / A_{2}\right) & =\lambda_{R}(F / B)+(r+1) \lambda_{R}(F / A) \\
& =\lambda_{R}\left(F_{2} / B_{2}\right)-\lambda_{R}\left(F_{1} B_{1} / B_{2}\right)+\lambda_{R}\left(F_{1} B_{1} / A_{1} B_{1}\right) \\
& =\lambda_{R}\left(F_{2} / A_{1} B_{1}\right) .
\end{aligned}
$$

In other words, $A_{2}=B_{1} A_{1}$. Thus, (v) implies (i). Finally, if (i) and (ii) are equivalent, then if (i) holds, (ii) and (iii) hold, and the proof of Theorem 4.1 shows that (iv) holds. Conversely, suppose that (iv) holds. Retaining our standard notation, it follows that the images of $C_{1}, \ldots, C_{r+1}$ in $\mathcal{A} / I \mathcal{A}$ form a regular sequence. Thus $B \mathcal{A} \cap I \mathcal{A}=I B \mathcal{A}$. Therefore $I B=I A \cap B$. By Corollary 2.3 (applied to $B$ ), $I A \subseteq B$, so $I A=I B$. By our assumption, (i) holds.

For part (ii), recall that a Cohen-Macaulay local ring $(S, n)$ is said to have minimal multiplicity if $e(S)=\mu(n)-\operatorname{dim}(S)+1$. Since $\mathcal{A}$ is Cohen-Macaulay if and only if the reduction number of $A$ is less than or equal to 1 , it remains to show that

$$
e\left(A_{\mathcal{P}}\right)=\mu\left(\mathcal{P} \mathcal{A}_{\mathcal{P}}\right)-\operatorname{dim}\left(\mathcal{A}_{\mathcal{P}}\right)+1 \text { if and only if } \mu(A)=\operatorname{ord}_{R}(A)+r .
$$

However, $\mu\left(\mathcal{P} \mathcal{A}_{\mathcal{P}}\right)=m+2$ and $\operatorname{dim}\left(\mathcal{A}_{\mathcal{P}}\right)=r+2$. Therefore, $\mathcal{A}_{\mathcal{P}}$ has minimal multiplicity if and only if $e\left(A_{\mathcal{P}}\right)=m-r+1$. On the other hand, let $B \subseteq A$ be a minimal reduction. Then $e\left(\mathcal{A}_{\mathcal{P}}\right)=e\left(\mathcal{B}_{\mathcal{Q}}\right)=\operatorname{ord}_{R}(J)+1$, where $J$ denotes the ideal of maximal minors associated to $B$. Note that the second equality follows, since by Proposition 1.1, $\mathcal{B}$ is a polynomial ring modulo an element having order $\operatorname{ord}_{R}(J)+1$. Since $\operatorname{ord}_{R}\left(I_{r}(A)\right)=\operatorname{ord}_{R}(J)$, the desired equivalence holds. The second statement in part (ii) now follows by invoking Theorem 2.1. 


\section{REFERENCES}

[BV] W. Bruns and U. Vetter, Determinantal Rings, Lecture Notes in Mathematics 1327, Springer-Verlag, Berlin and Heidelberg, 1988. MR 89i:13001

[BR] D. A. Buchsbaum and D.S. Rim, A generalized Koszul complex II. Depth and multiplicity, Trans. Amer. Math. Soc. 111 (1964), 197-224. MR 28:3076

[C1] S. D. Cutkosky, A new characterization of rational singularities, Inv. Math. 102 (1990), 157-177. MR 91h: 14045

[C2] S. D. Cutkosky, On unique and almost unique factorization of complete ideals II, Inv. Math. 98 (1989), 59-74. MR 90j:14016B

[H] C. Huneke, Complete ideals in two-dimensional regular local rings, Commutative Algebra, Proc. Microprogram, MSRI publication no. 15 (1989), 417-436. MR 90i:13020

[HS] C. Huneke and J. Sally, Birational extensions in dimension two and integrally closed ideals, J. Algebra 115 (1988), 481-500. MR 89e:13025

[Kap] I. Kaplansky, Commutative Rings, University of Chicago Press, 1974. MR 49:10674

[Ko] V. Kodiyalam, Integrally closed modules over two-dimensional regular local rings, Trans. Amer. Math. Soc. 347 (1995), 3551-3573. MR 95m:13015

[L] J. Lipman, Rational singularities with applications to algebraic surfaces and unique factorization, Inst. Hautes Etudes Sci. Publ. Math. 36 (1969), 195-279. MR 43:1986

[LT] J. Lipman and B. Teissier, Pseudo-rational local rings and a theorem of BriançonSkoda about integral closures of ideals, Michigan Math. Journal 28 (1981), 97-116. MR 82f: 14004

[R] D. Rees, Reduction of modules, Math. Proc. Camb. Phil. Soc. 101 (1987), 431-449. MR 88a: 13001

[Z] O. Zariski, Polynomial ideals defined by infinitely near base-points, Amer. J. Math. 60 (1938), 151-204.

[ZS] O. Zariski and P. Samuel, Commutative Algebra, vol. II, Van Nostrand Reinhold, New York, New York, 1960. MR 22:11006

Department of Mathematics, University of Kansas, Lawrence, Kansas 66045

E-mail address: dlk@math.ukans.edu

Current address: Vijay Kodiyalam, Institute of Mathematical Sciences, Tharamani, Madras 600 113, India

E-mail address: vijay@imsc.ernet.in 